$\$$

\title{
Quadrilateral Fuzzy Number
}

\author{
Pathinathan $\mathbf{T}^{1}$, Santhoshkumar $\mathbf{S}^{2}$ \\ ${ }^{1} P$. G. and Research Department of Mathematics, Loyola College, Chennai-34, India. \\ ${ }^{2} P$. G. and Research Department of Mathematics, Loyola College, Chennai-34, India. \\ *Corresponding author E-mail: pathinathan@gmail.com
}

\begin{abstract}
Fuzzy numbers are used to represent uncertainty. Various types of fuzzy numbers are used in practical applications. In this paper we define Perfect Pentagonal Fuzzy Number (PPFN), Quadrilateral Fuzzy Number (QNF) and Left skewed Quadrilateral Fuzzy Number and Right skewed Quadrilateral Fuzzy Number. We study their algebraic properties with numerical examples.
\end{abstract}

Keywords: fuzzy number, fuzzy operations, Perfect pentagonal fuzzy number (PPFN), quadrilateral fuzzy number (QFN), skewed fuzzy number.

\section{Introduction}

Lotfi A. Zadeh [6] introduced fuzzy sets in 1965 to quantify the uncertainty and vagueness of the qualitative words. The concept of fuzzy sets led researchers to define fuzzy numbers. Dubois and Prade [2], defined the fuzzy number as fuzzy subset of the real line [3]. The basic arithmetic structure of fuzzy numbers was developed by Dubois and Prade [1],[2], Mizumoto and Tanaka [8],[9], and Ma et al. [7]. The Triangular fuzzy number and Trapezoidal fuzzy numbers are most popular numbers used in the field of decision making [2],[4],[5]. T. Pathinathan and K. Ponnivalavan introduced Pentagonal Fuzzy Numbers [11] and Diamond Fuzzy numbers [10] in 2014. Also, T. Pathinathan and K. Ponnivalavan studied the Reverse Order Triangular, Trapezoidal and Pentagonal Fuzzy Numbers in 2014[12].

We define Perfect Pentagonal Fuzzy Number based on Pentagonal Fuzzy Number introduced by T. Pathinathan and K. Ponnivalavan [11]. We define Quadrilateral Fuzzy Number (QFN) as Left skewed Quadrilateral Fuzzy Number and Right skewed Quadrilateral Fuzzy Number followed by their Properties and arithmetic operations with numerical examples. The paper is organized as follows: Section two comprises the required preliminaries. In section three we define Perfect Pentagonal Fuzzy Number (PPFN). Also we define Quadrilateral Fuzzy Number (QFN) and explain with arithmetical illustrations. Section four describes conclusion.

\section{Preliminaries}

\subsection{Definition (Fuzzy Set) [6]}

A fuzzy set is characterized by a membership function mapping the element of a domain or space or universe of discourse $X$ to the unit interval, $[0,1]$. A fuzzy set $\underset{O_{-}}{A}$ in a universe discourse $X$ is defined as the following set pairs: $\underset{\%}{A}=\left\{x, m_{\%} ; x \dot{\sim} X\right\}$. $m_{\frac{A}{\%}}: X ®[0,1]$ is a mapping called the degree of membership function of the fuzzy set $m_{A}(x)$ is called the membership value of $x \dot{\tau} X$ in the fuzzy set $\underset{o \text {. }}{A}$. These membership grades are often represented by real numbers ranging from $[0,1]$.

\subsection{Definition (Fuzzy Number) [2],[3]}

A fuzzy set set $A_{0 t}$, defined on the set of real numbers $R$ is said to be fuzzy number, if it satisfies the following characteristics.
a) $\quad A_{0}$ is normal
b) $A_{0,}^{A}$ is convex set and
c) The support of $\underset{O O_{0}}{A}$ is closed and bounded.

\subsection{Definition (Triangular Fuzzy Number) [5]}

A fuzzy set $\underset{\%}{A}=(a, b, c)$, defined on the set of real numbers $R$ is said to be Triangular Fuzzy Numbers, when its membership function is given as below.

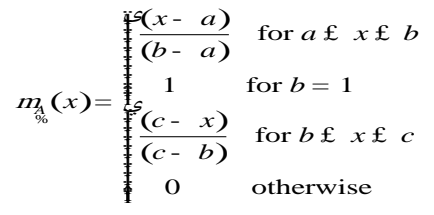

where $a £ b £ c$.

\subsection{Definition (Trapezoidal Fuzzy Number) [4]}

Trapezoidal Fuzzy Number is defined as $\underset{\%}{A}=(a, b, c, d)$, where all $a, b, c$ and $d$ are real numbers and its membership function is given below.

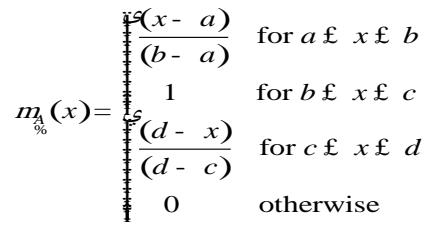

where $a £ b £ c £ d$. 


\subsection{Definition (Pentagonal Fuzzy Number) [11]}

A Pentagonal Fuzzy Number (PNF) of a fuzzy set $\underset{o \text { t. }}{A}$ is defined as $\underset{f_{p}}{A_{p}}=(a, b, c, d, e)$ and its membership function is given below,

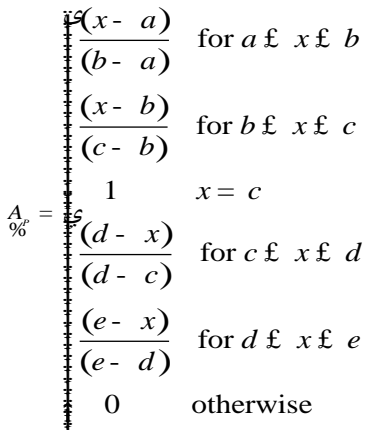

and $A_{o p}$ should satisfy the following conditions:

1) $\quad m_{\substack{A_{P} \\ \sigma_{p}}}(x)$ is a continuous functions in the interval $[0,1]$.

2) $\quad m_{\substack{A_{p} \\(x)}}(x)$ is strictly increasing and continuous function on $[a, b]$ and $[c, d]$

3)

$m_{A_{f}}(x)$ is strictly decreasing and continuous on $[c, d]$ and $[d, e]$.

\section{Quadrilateral Fuzzy Number (QFN)}

A Quadrilateral Fuzzy Number is either Left skewed Quadrilateral Fuzzy Number (LsQFN) or Right skewed Quadrilateral Fuzzy Number (RsQFN).

\subsection{Perfect Pentagonal Fuzzy Number}

\subsubsection{Definition}

A Pentagonal Fuzzy number is defined as Perfect Pentagonal Fuzzy Number if the following conditions are satisfied along with the existing conditions. We find the slope of line segments corresponding to the values between $[a, b],[b, c],[c, d]$ and $[\mathrm{d}, \mathrm{e}]$; say $m_{1}, m_{2}, m_{3}$ and $m_{4}$. i.e., Slope of the line segments are $\frac{y_{2}-y_{1}}{x_{2}-x_{1}}=m_{1}, \frac{y_{3}-y_{2}}{x_{3}-x_{2}}=m_{2}, \frac{y_{4}-y_{3}}{x_{4}-x_{3}}=m_{3}$ and $\frac{y_{5}-y_{4}}{x_{5}-x_{4}}=m_{4}$.

1) If $m_{1}=m_{2}$ and $m_{3}=m_{4}$, then this fuzzy number becomes a Triangular Fuzzy Number.

2) If $m_{1}{ }^{1} m_{2}$ and $m_{3}{ }^{1} m_{4}$, then this fuzzy number is called a Perfect Pentagonal Fuzzy Number (PPFN).

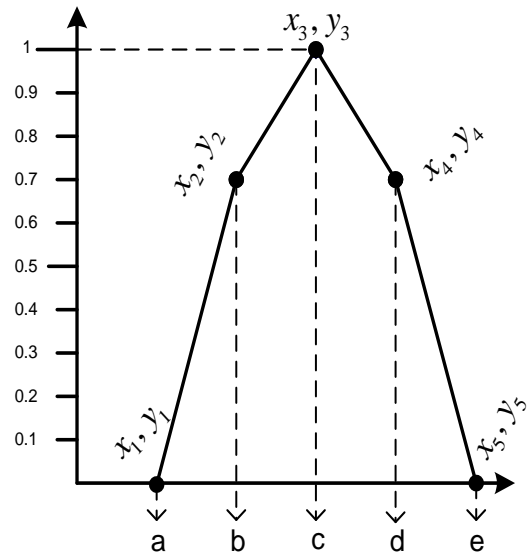

Fig. 1: Perfect Pentagonal Fuzzy Number.

\subsection{Right skewed Quadrilateral Fuzzy Number}

In Perfect Pentagonal Fuzzy Number, if $m_{1}=m_{2}$ and $m_{3}{ }^{1} m_{4}$, then it becomes Right skewed Quadrilateral Fuzzy Number (RsQFN). It defines as follow:

\subsubsection{Definition}

Right skewed Quadrilateral Fuzzy Number ${\underset{\sigma}{O_{R}}}_{Q_{R}}=(a, b, c, d)$ on the set of real numbers $R$ is defined as follows:

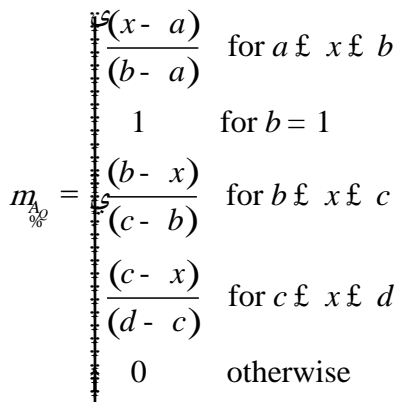

and $\underset{O O}{A}$ should satisfy the following conditions:

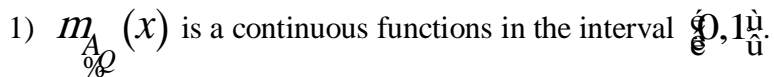

2) $m_{A_{Q}}(x)$ is strictly increasing and continuous function on éc, $b_{\text {ù }}^{\text {ù }}$

3) $m_{\mathscr{A}}(x)$ is strictly decreasing and continuous function on

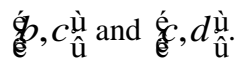

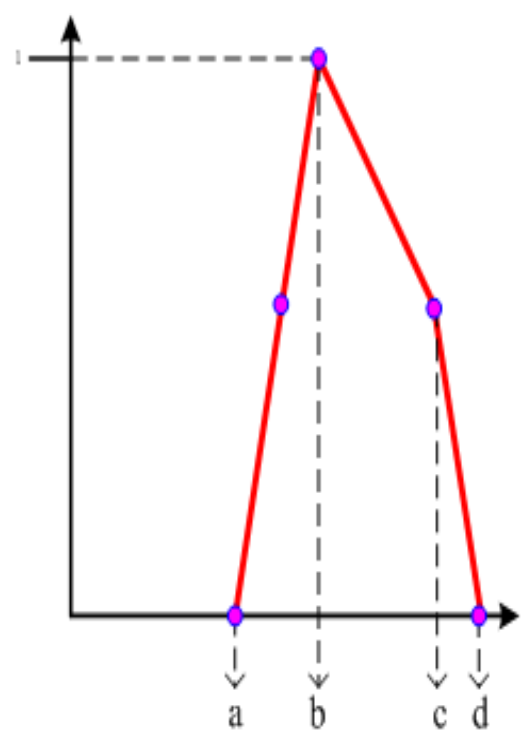

Fig. 2: Right skewed Quadrilateral Fuzzy Number.

\subsection{Left skewed Quadrilateral Fuzzy Number}

In Perfect Pentagonal Fuzzy Number, $m_{1}{ }^{1} m_{2}$ and $m_{3}=m_{4}$, then it becomes Left skewed Quadrilateral Fuzzy Number (LsQFN). It defines as follow:

\subsubsection{Definition}

Left skewed Quadrilateral Fuzzy Number ${\underset{o}{\rho_{L}}}_{C_{L}}=(a, b, c, d)$ on the set of real numbers $R$ is defined as follows: 


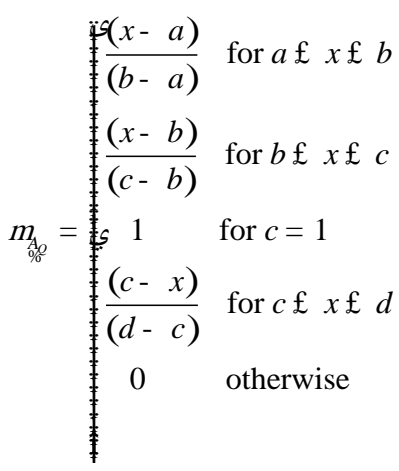

and $\underset{O O}{A}$ should satisfy the following conditions:

1) $m_{\frac{A}{Q}}(x)$ is a continuous functions in the interval $q$ é 1 ù

2) $m_{\mathscr{Q} Q}(x)$ is strictly increasing and continuous function on

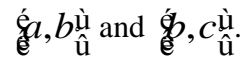

3) $m_{A_{Q}}(x)$ is strictly decreasing and continuous function on ććc, $d_{\hat{\mathrm{u}}}^{\mathrm{ù}}$

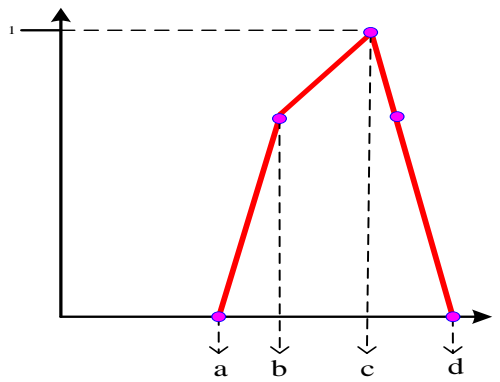

Fig. 3: Left skewed Quadrilateral Fuzzy Number.

3.4. Properties on Left skewed Quadrilateral Fuzzy Number (LsQFN)Left skewed Quadrilateral Fuzzy Number

\subsubsection{Union}

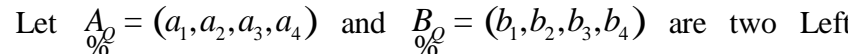
skewed Quadrilateral Fuzzy Numbers, then the union of the LsQFNs is, $\underset{\%}{m_{\%} \cup B} \in\left\{\left(a_{1}+b_{1}\right),\left(a_{2}+b_{2}\right),\left(a_{3}+b_{3}\right),\left(a_{4}+b_{4}\right)\right\}$. The

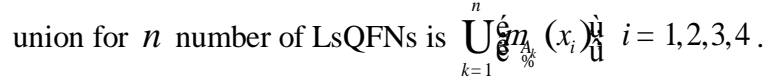

\subsubsection{Intersection}

Similarly the intersection for $n$ LsQFNs is

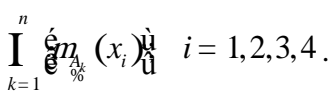

\subsubsection{Complement}

Let $A_{\mathscr{O}}^{A}=\left(a_{1}, a_{2}, a_{3}, a_{4}\right)$ be a LsQFN, then the complement of $A_{\mathscr{O}}^{A}$ is, $\bar{o}_{O O}=1-\underset{O O}{A_{O}}$. i.e. $\bar{A}_{O O}=\left(1-a_{1}, 1-a_{2}, 1-a_{3}, 1-a_{4}\right)$.

Note: Properties on Right skewed Quadrilateral Fuzzy Number (RsQFN) are same as LsQFN.
3.5. Arithmetic operations on Left skewed Quadrilateral Fuzzy Number (LsQFN)

\subsubsection{Addition}

Let $A_{o f}=\left(a_{1}, a_{2}, a_{3}, a_{4}\right)$ and $\underset{O Q}{B}=\left(b_{1}, b_{2}, b_{3}, b_{4}\right)$ are two Left skewed Quadrilateral Fuzzy Numbers (LsQFN), then the addition of two LsQFN is, $m_{a_{g}+B_{g, q}}=\left(\left(a_{1}+b_{1}\right),\left(a_{2}+b_{2}\right),\left(a_{3}+b_{3}\right),\left(a_{4}+b_{4}\right)\right)$.

\subsubsection{Subtraction}

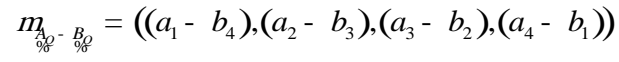

\subsubsection{Scalar Multiplication}

$1 \times \underset{O O}{A}=1 \times\left(a_{1}, a_{2}, a_{3}, a_{4}\right)=\left(1 \times a_{1}, 1 \times a_{2}, I \times a_{3}, I \times a_{4}\right)$

\subsubsection{Multiplication}

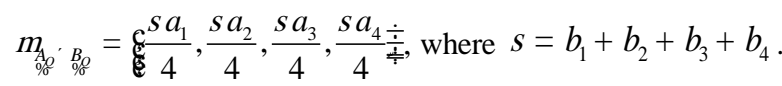

\subsubsection{Division}

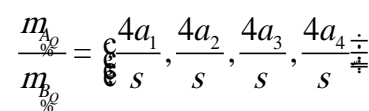

Note: Arithmetic operations on Right skewed Quadrilateral Fuzzy Number (RsQFN) are same as LsQFN.

\subsection{Numerical example of Arithmetic operations on LsQFN}

Let $m_{d, q}=(0.3,0.4,0.7,0.8)$ and $m_{g, q}=(0.5,0.7,0.8,0.9)$ are two Left skewed Quadrilateral Fuzzy Numbers (LsQFNs).

\subsubsection{Addition}

$$
\begin{aligned}
m_{\substack{\mathrm{d} / R+B g \\
g / g}} & =((0.3+0.5),(0.4+0.7),(0.7+0.8),(0.8+0.9)) \\
& =(0.8,1.1,1.5,1.7)
\end{aligned}
$$

\subsubsection{Subtraction}

$$
\begin{aligned}
& m_{\substack{\mathrm{A}-\frac{B}{g} \\
g / g}}=((0.3-0.9),(0.4-0.8),(0.7-0.7),(0.8-0.5)) \\
& =(-0.6,-0.4,0,0.3)
\end{aligned}
$$

\subsubsection{Scalar Multiplication}

$$
\begin{aligned}
\underset{\mathscr{O} A_{0}}{2 \times A_{0}} & =2 \times(0.3,0.4,0.7,0.8) \\
& =\left(2^{\prime} \quad 0.3,2^{\prime} \quad 0.4,2^{\prime} 0.7,2^{\prime} 0.8\right) \\
& =(0.6,0.8,1.4,1.6)
\end{aligned}
$$

\subsubsection{Multiplication}

$$
\begin{aligned}
& s=0.5+0.7+0.8+0.9=2.9 \text {; }
\end{aligned}
$$

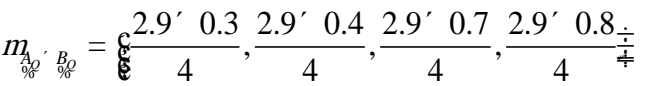

$$
\begin{aligned}
& =(0.2175,0.29,0.5075,0.58)
\end{aligned}
$$




\subsubsection{Division}

$$
\begin{aligned}
\frac{m_{A_{Q}}}{m_{B_{Q}}} & =\frac{4^{\prime} 0.3}{2.9}, \frac{4^{\prime} 0.4}{2.9}, \frac{4^{\prime} 0.7}{2.9}, \frac{4^{\prime} 0.8}{2.9} \frac{\dot{\dagger}}{\frac{\dot{t}}{\div}} \\
& =(0.4137,0.5517,0.9655,1.1034)
\end{aligned}
$$

\section{Conclusion}

We defined Perfect Pentagonal Fuzzy Number (PPFN). We have introduced and defined Left and Right Skewed Quadrilateral Fuzzy Number (QNF). We propose its algebraic Properties with numerical examples. Quadrilateral Fuzzy Number can be applied to any problem which has four points in representation with one value as normality. This number might be representing various research problems in future.

\section{References}

[1] Dubois D \& Prade H (1987), Fuzzy number: An overview, In the Analysis of Fuzzy Information Volume 1: Mathematics, CRC Press, Boca Flaton, FL, pp:3-39.

[2] Dubois D \& Prade H (1978), Operations on fuzzy numbers. International Journal of Systems Science, Vol.9, No.6, pp:613-626.

[3] Klir GJ (2000), Fuzzy Sets: An Overview of Fundamentals, Applications and Personal views, Beijing Normal University Press, China, pp:44-49.

[4] Zimmermann HJ (1996), Fuzzy Set Theory and its Applications, 3 rd edn., Kluwer Academic Publishers, Boston, Massachusetts.

[5] Kauffmann \& Gupta M (1980), Introduction to Fuzzy Arithmetic, Theory and Applications, Van Nostrand Reinhold, New York.

[6] Zadeh LA (1965), Fuzzy sets. Information and Control, Vol.8, pp:338-353.

[7] Ma M, Friedman M \& Kandel A (1999), A new fuzzy arithmetic Fuzzy Sets and Systems, Vol.108, pp:83-90.

[8] Mizumoto M \& Tanaka K (1979), Some Properties of fuzzy numbers. In Advances in Fuzzy Set Theory and Applications, NorthHolland, Amsterdam, pp:156-164.

[9] Mizumoto M \& Tanaka K (1976), The four operations of arithmetic on fuzzy numbers. Systems Comput. Controls, Vol.7, No.5, pp:7381.

[10] Pathinathan T, \& Ponnivalavan K (2014), Diamond fuzzy numbers Journal of Fuzzy set Valued Analysis, Vol.1, pp.36-44.

[11] Pathinathan T, \& Ponnivalavan K (2014), Pentagonal fuzzy numbers. International Journal of Computing Algorithm, Vol.3, pp:1003-1005.

[12] Pathinathan T \& Ponnivalavan K (2014), Reverse Order Triangular, Trapezoidal and Pentagonal Fuzzy Numbers. Annals of Pure and Applied Mathematics, Vol.8, pp:51-58. 